\title{
BOTDA sensor network with power by light remote switching
}

\author{
A. Ullán*a , M. Bravo ${ }^{b}$, A. Zornoza ${ }^{b}$, A. Loayssa ${ }^{b}$, M. Lopez-Amo ${ }^{\text {b }}$ J.M. Lopez-Higuera ${ }^{\text {a }}$ \\ ${ }^{a}$ Photonics engineering group, Dpto. TEISA, Universidad de Cantabria, av. Los Castros s/n, \\ Santander, Cantabria, Spain. \\ ${ }^{\mathrm{b} O p t i c a l ~ c o m m u n i c a t i o n s ~ g r o u p, ~ D p t o . ~ d e ~ I n g e n i e r i ́ a ~ E l e ́ c t r i c a ~ y ~ E l e c t r o ́ n i c a, ~ U n i v e r s i d a d ~ P u ́ b l i c a ~}$ \\ de Navarra, Campus Arrosadía, Pamplona, Navarra, Spain.
}

\begin{abstract}
We present and demonstrate a Brillouin Optical Time Domain Analysis (BOTDA) based long range sensor network with remote switching. Two different $5 \mathrm{~km}$ long sections were monitored alternatively by using a fast remotely controlled and optically powered up optical switch. The sensed fibers were located $10 \mathrm{~km}$ away from the interrogation unit. The BOTDA unit uses a simplified configuration to reduce the sensor network costs. Proof-of-concept experiments were carried out verifying the capacity of the proposed system.
\end{abstract}

Keywords: BOTDA sensor, remote sensing, optical switch, sensor network

\section{INTRODUCTION}

Optical fiber sensors are nowadays one of the most promising technologies for structure health monitoring. Fiber Bragg Grating (FBG) based sensors and Reflectometry based sensors, namely OTDR systems, have been the most commonly implemented techniques up to now [1]. Nevertheless, BOTDA systems are especially interesting since they are able to measure temperature and/or strain in a distributed manner all along the structure. The spatial resolution is typically limited to $1 \mathrm{~m}$ due to the phonon lifetime [2], but a number of novel techniques have been presented recently in order to attain sub-meter spatial resolutions [3,4,5]. Long range BOTDA systems have also been presented, attaining measurement of tens of kilometres with $1 \mathrm{~m}$ resolution, or even longer distances when it is combined with Raman amplification along the channel [6]. However, the cost and the complexity of the setup is the main issue slowing down wide world deployment of this technology.

Multiplexing sensors is the most reasonable technique in order to reduce the cost of operation and increase the performance of the network. Sometimes different kind of sensors can also be combined and multiplexed, enhancing the sensing capabilities of the system [7]. The use of optical switches is crucial for reducing the cost of structure health monitoring, since they allow sensing many structures by means of a single interrogation unit. They can also be used to enhance the robustness of the network against fiber breaks. Furthermore, optical powering is a key issue for a new generation of intelligent sensor networks [8] with a broad range of monitoring applications.

In this paper, we present a sensor network appropriate to monitor multiple structures with one single simplified BOTDA system. It uses a power by light optical switch for selecting the structure to be sensed. This switch is controlled remotely and optically, so no power supplies are needed away from the interrogation station. The BOTDA configuration used to interrogate the sensor network is simplified with respect to typical BOTDA setups. It uses a highly nonlinear fiber to generate a first Brillouin scattering Stokes wave that will be used to generate the pump pulses [9]. Wideband electrooptic modulators (EOM) and microwave synthesized generators are then not needed, decreasing the cost of the setup.

\section{DESCRIPTION OF THE SENSOR NETWORK}

The proposed sensor network is depicted in figure 1. It consists of a BOTDA system used to interrogate a $25 \mathrm{~km}$ long fiber channel, where the central $5 \mathrm{~km}$ are connected trough two optical switches, where the structures to be sensed are 
intended to be. A Raman pump at $1445 \mathrm{~nm}$ is necessary to remotely feed a photoelectric cell that supplies the power to the switch. Another optical signal is introduced in the fiber line to remotely control the switch position.

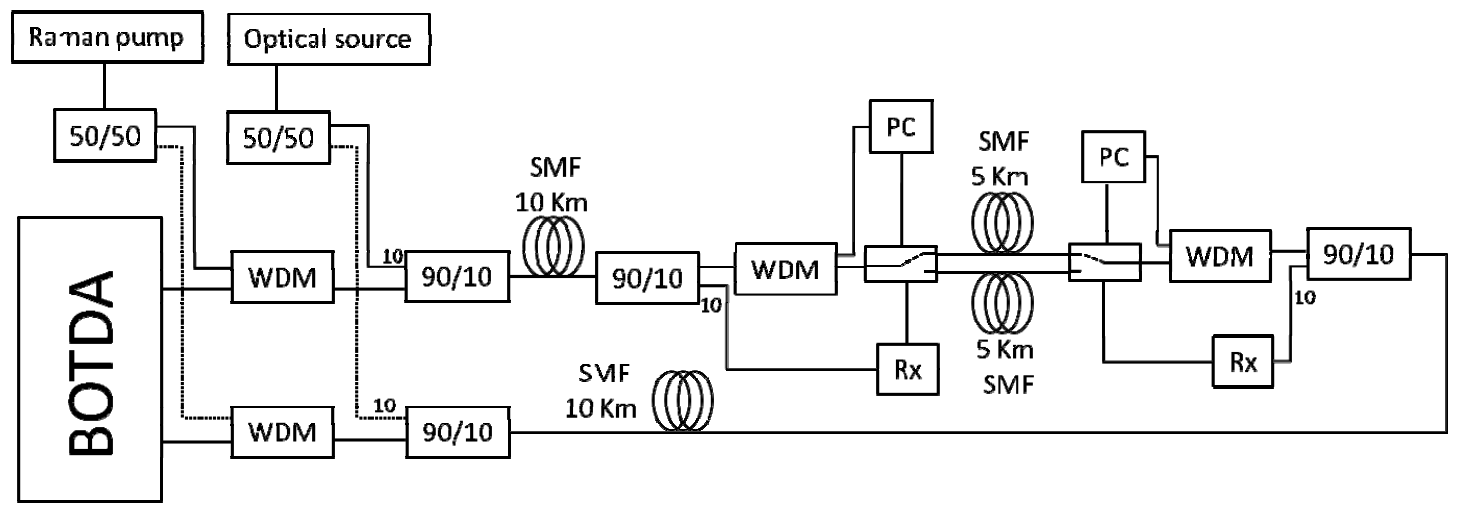

Figure 1.Structure of the BOTDA sensor network. WDM: wavelength division multiplexer; PC: photoelectric cell; Rx: photoreceiver.

We used a photoelectric cell to drive a commercial, low consumption fiber optic switch with MEMS technology $1 \times 2$ developed by DiCon Fiberoptics Inc. The power converter works at $1445 \mathrm{~nm}$, converting $125 \mathrm{~mW}$ of optical power to $\sim 60 \mathrm{~mW}$ of electrical power. In order to select the channel, two different voltages are necessary on the electrical ports of the switch. As the photoelectric cell provides a maximum of 4.8V, DC-DC voltage converters are necessary to drive de optical switch as shown in figure 2(a). An optical control system has been developed so that channel 1 is selected when an optical intensity above $0.001 \mathrm{~mW}$ (between $1310 \mathrm{~nm}$ and $1550 \mathrm{~nm}$ ) illuminates the PIN detector. Otherwise, channel 2 is active. For measuring the switch response, the light coupled at the input port was detected and monitored by an oscilloscope. Figure 2(b) shows the rising flank of the switch response. A switching time less than $2 \mathrm{~ms}$ is observed.

(a)

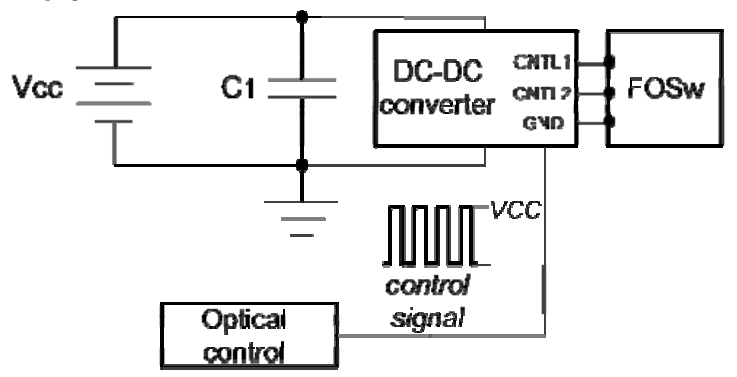

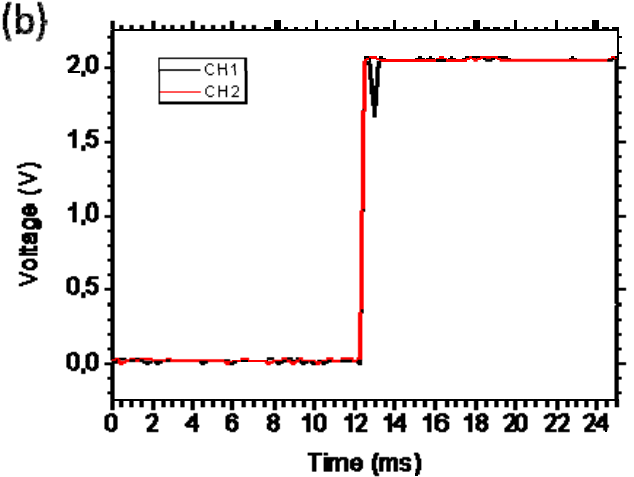

Figure 2. (a) Electronics setup for the remote control of the fiber optic switch. (b) Zoom of the switch rising flank response for both channels.

The BOTDA system is described in figure 3. It operates in the so called loss regime. The laser output (10dBm) is divided in two branches. The lower one carries the continuous probe wave through an optical isolator and a variable attenuator used to adjust the power entering the fiber under test (FUT). The upper one amplifies the laser output to induce a new downshifted signal inside a $5 \mathrm{~km}$ long highly nonlinear fiber (HNF) by means of Brillouin scattering. Notice that the EDFA before the circulator would not be needed if a high power CW DFB laser were deployed, further reducing the setup costs. The first downshifted photons are generated through the spontaneous Brilloiun scattering process and then, they are amplified by stimulated Brillouin scattering all along its way back to the circulator. The Brillouin frequency shift $\left(v_{\mathrm{B}}\right)$ for this fiber is $9.992 \mathrm{GHz}$, while the standard fibers used to monitor the structures (the FUT) have $v_{\mathrm{B}}$ around $10.8 \mathrm{GHz}$. This frequency shifted signal comes out from the circulator and is modulated in the MHz range by an EOM. Pulsing of the signal is done in the RF domain by means of an RF switch and a pulse generator [10]. The amplitude of the pulses is adjusted and its polarization scrambled in order to avoid modulation instability and polarization dependence respectively in our measurements. The BOTDA signal comes out from port 3 of the second circulator shown in the 
scheme, and it is filtered out and boosted up prior to be captured by a low noise photodetector. Then, it is analyzed in an oscilloscope. The oscilloscope and the RF generator are computer controlled in order to sweep the modulation frequency around the Brillouin gain curve of the FUT. This configuration allows using a low-speed EOM with relatively low extinction ratio (ER) as well as a low frequency RF generator (in the MHz range), avoiding the use of costly wide-band specialty high-ER EOM and microwave synthesized generators.

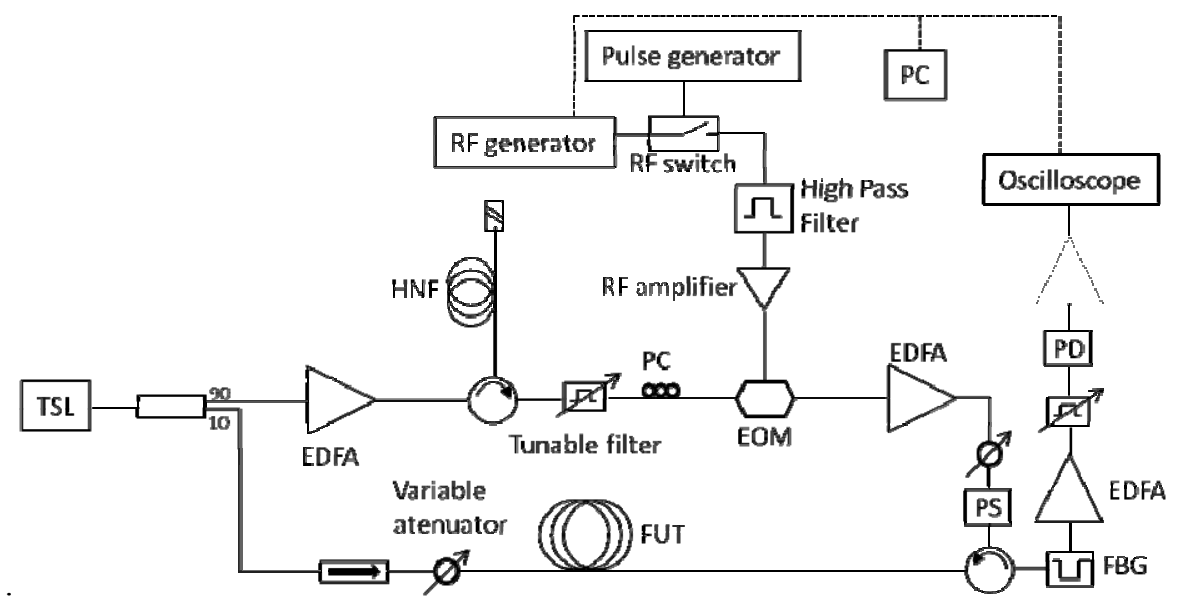

Figure 3.Scheme of the BOTDA setup used for our measurements. TSL: tunable semiconductor laser; PC: polarization controller; PD: photodetector; HNF: highly nonlinear fiber; PS: polarization scrambler; FUT: fiber under test

\section{EXPERIMENTAL DEMONSTRATION}

In order to check the capacity of the sensor network, proof -of-concept experiments were carried out in our laboratory. $400 \mathrm{~mW}$ of Raman pump were launched into the fiber $(200 \mathrm{~mW}$ on each branch) to feed up the photoelectric cells that act as a power supply for the optical switches. This Raman signal also contributes to compensate the optical losses of the sensing line due to the induced distributed Raman gain along the channel, although the gain with this pump power stays low. Another optical signal was needed to remotely control the switch position. We used the output of a tunable laser source, tuned at $1546 \mathrm{~nm}$, but any stable light source is appropriate as long as the receiver gets at least $-28 \mathrm{dBm}$ at any wavelength between 1310 and $1550 \mathrm{~nm}$. The power of the continuous probe wave was $-8 \mathrm{dBm}$, and the power of the pump pulses was $24.4 \mathrm{dBm}$ at the entrance of the network. The pulses were $20 \mathrm{~ns}$ long, so the measurements have $2 \mathrm{~m}$ as spatial resolution. $1 \mathrm{~m}$ resolution measurements were also carried out but the results were much noisier, so a fine adjustment of the whole system would have to be done to reduce optical losses to the minimum and adapt the optical powers of the pump and probe waves as well as the Raman signal to the optimal configuration for compensating the network losses.

In the section to be monitored, between the switches, we placed 2 fiber spools of $5 \mathrm{~km} \mathrm{SMF}$. At the end of one of them, we added $180 \mathrm{~m}$ of the same fiber. The temperature of this added section was kept constant at $60^{\circ} \mathrm{C}$. Figure 4(a) shows the value of $v_{B}$ for each sensed branch. We can clearly distinguish the two $10 \mathrm{~km}$ sections before and after the $5 \mathrm{~km}$ spools. Note that de second section of $10 \mathrm{~km}$ is in fact the ensemble of two spools of 6 and $4 \mathrm{~km}$ respectively, spliced together, with the correspondent optical losses and inhomogeneity in this region. We observe in red the results obtained for the spool to which we added $180 \mathrm{~m}$ of heated SMF. We clearly see that $v_{\mathrm{B}}$ for this region has moved up to 10.852 $\mathrm{GHz}, 39 \mathrm{MHz}$ higher than $v_{\mathrm{B}}$ for the previous $5 \mathrm{~km}$, kept at room temperature. This agrees with the well known $v_{\mathrm{B}}$ shift ratio in $\mathrm{SMF}$ of $1.15 \mathrm{MHz} /{ }^{\circ} \mathrm{C}$ [11]. The difference observed between the two measurements in the $5 \mathrm{~km}$ section $\left(v_{\mathrm{B}}\right.$ varies from 10.808 to $10.813 \mathrm{MHz}$ ) is due to slight differences in the two spools, i.e. different winding tension. The temperature resolution measured in the $5 \mathrm{~km}$ was estimated to be near $2.8^{\circ} \mathrm{C}$. In figure $4(\mathrm{~b})$ we observe the Brillouin spectra along the $5 \mathrm{~km}+180 \mathrm{~m}$ section. It can be seen that the Brillouin gain curve shifts to lower frequencies when entering the $5 \mathrm{~km}$ section from the $10 \mathrm{~km}$ SMF channel, and moves up in frequency for the heated $180 \mathrm{~m}$ long section. 

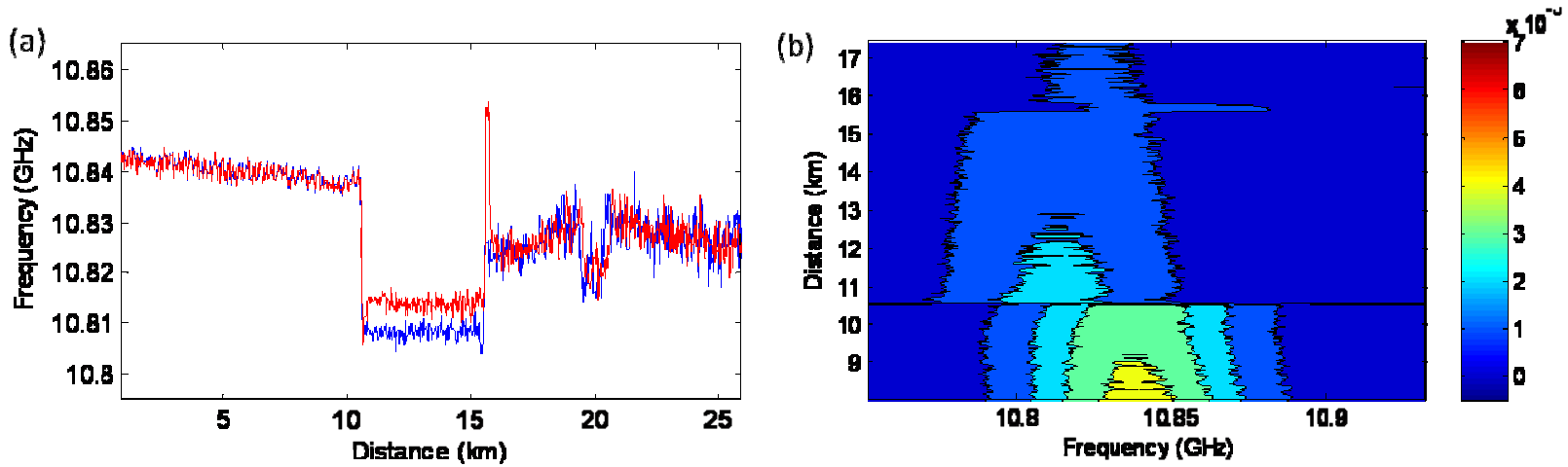

Figure 4.(a)Brillouin frequency shift along the sensing channel.(b)Brillouin spectra along the sensing channel focused on the 5 $\mathrm{km}$ section with $180 \mathrm{~m}$ heated to $60^{\circ} \mathrm{C}$.

\section{CONCLUSIONS}

A new optical sensor network based on BOTDA technology has been presented and experimentally validated. A fast remotely and optically controlled power by light optical switch has been used to select the structure to be sensed, $10 \mathrm{~km}$ away from the interrogation unit. We performed temperature distributed measurements along 2 different sections of $5 \mathrm{~km}$ at $2 \mathrm{~m}$ resolution. The BOTDA unit employed to interrogate the structures uses a simplified configuration that allows avoiding wide-band high-ER specialty EOMs and high frequency microwave synthesized generators.

\section{ACKNOWLEDGEMENTS}

This work was supported by the Spanish Government project TEC2010-20224-C02.

\section{REFERENCES}

[1] J. M. Lopez-Higuera, L. Rodriguez-Cobo, A. Quintela, A. Cobo, "Fiber optic sensors in structural health monitoring", Journal of Ligthwave Technology, Vol. 29, No. 4, pp. 587-608, 2011.

[2] V. P. Kalosha, E. A. Ponomarev, L. Chen and X. Bao, "How to obtain high spectral resolution of SBS-based distributed sensing by using nanosecond pulses”, Optics Express, vol. 14, No. 6, pp. 2071-2078, 2006.

[3] A. W. Brown, B. G. Colpitts and K. Brown, "Distributed sensor based on dark-pulse Brillouin scattering", Photonics Technology Letters, IEEE, vol. 17, pp. 1501-1503, 2005.

[4] W. Li, X. Bao, Y. Li and L. Chen, "Differential pulse-width pair BOTDA for high spatial resolution sensing", Optics express, vol. 16, pp. 21616-21625, 2008.

[5] S.M. Foaleng, M. Tur, J. C. Beugnot and L. Thévenaz, "High spatial and spectral resolution long range sensing using Brillouin echoes", Journal of Lightwave Technology, vol. 28, pp. 2993-3003, 2010.

[6] M. A. Soto, G. Bolognini and F. Di Pasquale, "Optimization of long-range BOTDA sensors with high resolution using first-order bi-direction Raman amplification", Optics Express, Vol. 19, No. 5, pp. 4444-4457, 2011.

[7] A. Zornoza, R. A. Pérez-Herrera, C. Elosúa, S. Diaz, C. Bariain, A. Loayssa, M. Lopez-Amo, "Long-range hybrid network with point and distributed Brillouin sensors using Raman amplification," Optics Express, Vol. 18, No. 9, pp. 9531-9541, 2010.

[8] R. Peña, C. Algora, I. R. Matias, and M. López-Amo, "Fiber-based 205-mW (27\% efficiency) power-delivery system for an all-fiber network with optoelectronic sensor units", Applied Optics, Vol. 38, No. 12, pp 2463-2466, 1999.

[9] A. Zornoza and A. Loayssa, "Low-cost Brillouin optical time domain analysis (BOTDA) distributed sensor setup", Proc. SPIE 7653, 765334, 2010;

[10] A. Zornoza, D. Olier, M. Sagues and A. Loayssa, "Brillouin distributed sensor using RF shaping of pump pulses", Measurement Science and Technology, vol. 21, No. 9, 094021, doi:10.1088/0957-0233/21/9/094021, 2010.

[11] J. M. Lopez-Higuera. Handbook of optical fibre sensing technology, Wiley \& Sons, 2002. 